Revue

Revue de l'histoire des religions

de Ihistoire des religions

1 | 2016

Varia

\title{
Viktória HEDVIG DEÁK, La légende de sainte Marguerite de Hongrie et l'hagiographie dominicaine
}

Paris, Éditions du Cerf (« Histoire »), 2013

Patrick Henriet

\section{(2) OpenEdition}

Journals

Édition électronique

URL : http://journals.openedition.org/rhr/8515

DOI : 10.4000/rhr.8515

ISSN : 2105-2573

Éditeur

Armand Colin

Édition imprimée

Date de publication : 1 mars 2016

Pagination : 124-126

ISBN : 978-2-200-93059-2

ISSN : 0035-1423

Référence électronique

Patrick Henriet, « Viktória HEDVIG DEÁk, La légende de sainte Marguerite de Hongrie et l'hagiographie dominicaine », Revue de l'histoire des religions [En ligne], 1 | 2016, mis en ligne le 05 avril 2016, consulté le 24 septembre 2020. URL : http://journals.openedition.org/rhr/8515 ; DOI : https://doi.org/10.4000/ rhr.8515

Ce document a été généré automatiquement le 24 septembre 2020

Tous droits réservés 


\section{Viktória HEDVIG DEÁK, La légende de sainte Marguerite de Hongrie et l'hagiographie dominicaine}

Paris, Éditions du Cerf (« Histoire »), 2013

\section{Patrick Henriet}

\section{RÉFÉRENCE}

Viktória HEDVIG DEÁK, La légende de sainte Marguerite de Hongrie et l'hagiographie dominicaine, Paris, Éditions du Cerf (« Histoire »), 2013, 23,5 cm, 351 p., $39 €$, ISBN 978-2-204-09685-0.

1 Élisabeth de Hongrie (†1231) est célèbre, en particulier grâce à Montalembert qui publia sa Vie en 1836. Sa nièce Marguerite de Hongrie l'est moins (de même d'ailleurs qu'Élisabeth de Portugal, morte en 1336 et petite nièce d'Élisabeth de Hongrie). Fille du roi Béla IV, elle entre à dix ans chez les dominicaines de l'île des Lièvres à Budapest (actuelle île Marguerite). Elle y meurt en 1270, à 28 ans. À partir d'une date discutée, mais en tout cas dès la première moitié $d u X \mathrm{XI}^{\mathrm{e}}$ siècle, elle eut la réputation d'avoir reçu les stigmates et sa notoriété fut telle qu'une version italienne du Miroir des simples âmes de Marguerite Porete lui est attribuée. Dominicaine de la congrégation de SainteMarguerite de Hongrie, Viktória Hedvig Deák reprend dans ce livre les principaux éléments du dossier hagiographique : précisons cependant qu'il ne s'agit pas d'initier le lecteur à un culte peu connu en France mais plutôt d'étudier sa place dans la production hagiographique dominicaine. L'auteure se situe dans la continuité d'une histoire de la spiritualité et des modèles de sainteté profondément marquée par André Vauchez.

2 Pour comprendre ce qui suit, il convient d'abord de présenter brièvement les diverses pièces du dossier. Nous le ferons en précisant chaque fois leur numéro dans la Bibliotheca Hagiographica Latina (BHL), ce qui n'est malheureusement jamais fait dans le 
livre. Après la mort de Marguerite en 1270, une première enquête visant la canonisation fut lancée sous la direction de l'archevêque d'Esztergom. Il n'en reste aucune trace, mais le pape rouvrit l'enquête en 1276: on a donc cette fois-ci les dépositions de cent-dix témoins, dont trente-huit religieuses (BHL 5330). Peu de temps auparavant, vers 1275, un auteur anonyme (sans doute Marcel, le confesseur de Marguerite) avait composé une Legenda vetus (BHL 5330d) qui ne fut découverte qu'en 1937. Les deux pièces suivantes, Legenda minor (BHL 5332) et Legenda maior (BHL 5331) datent du début des années 1340. Grâce aux travaux de Gábor Klaniczay, l'auteure confirme que la Legenda minor dépend de la Legenda maior, qu'elle résume. L'auteur du texte le plus court est nommé dans l'un des manuscrits : c'est Garin de Gy-l'Évêque (Yonne), qui écrit à Avignon sur la demande du général dominicain Hugues de Vaucemain. Maître en théologie, Garin fit l'essentiel de sa carrière entre Paris et Avignon. Il termina maître général de l'ordre et mourut de la peste en 1348. À la suite de Gábor Klaniczay, VHD propose de lui attribuer aussi la Legenda maior. Cette solution, probable, ne peut être démontrée. Quoi qu'il en soit, au début des années 1340, au sommet même de l'ordre dominicain, Marguerite de Hongrie permit de proposer un idéal de sainteté militant et orthodoxe (ce qui avait été recommandé par les chapitres généraux de Limoges en 1334 et de Londres en 1335).

La première partie du livre est une sorte d'histoire de l'hagiographie dominicaine qui passe en revue les principaux textes depuis la mort de Dominique et s'arrête en particulier sur la question de la sainteté féminine chez les dominicains. Marguerite de Hongrie, même si elle ne fut finalement canonisée qu'en 1943, est la première sainte femme clairement reconnue par l'ordre. L'auteure connait bien les dossiers masculins et féminins, en particulier pour la Hongrie et l'Italie. Parmi « les saints dont la légende contemporaine ne nous est pas parvenue ", il faut rectifier ce qui est dit p. 115 de Pedro González ( $† 1246)$, saint Elme: on ne peut se contenter de rappeler que «les Acta sanctorum affirment l'existence d'une légende, mais reconnaissent ne pas pouvoir la trouver » : le texte de la Vita (tronqué) et de 126 miracles, rédigés peu après la mort du saint, ont été édités par Enrique Flórez dès 1767 (España Sagrada, 23, p. 245-285). Une deuxième partie étudie la Legenda Maior (c'est le vrai sujet du livre) sous l'angle des coordonnées hagiographiques: manuscrits, contexte de rédaction et de diffusion, auteur, rapports avec les autres pièces du dossier. On appréciera tout particulièrement les pages qui montrent comment ce texte fut diffusé par et dans les milieux observants au début $\mathrm{du} x \mathrm{x}^{\mathrm{e}}$ siècle, particulièrement grâce à Thomas de Sienne (1434), prieur à Venise et grand artisan du culte de Catherine de Sienne (1380). Ce n'est guère avant la seconde moitié du XIV ${ }^{\mathrm{e}}$ siècle que les dominicains promurent les saintes femmes issues de leur ordre, particulièrement en Italie. Les efforts déployés en faveur de Marguerite de Hongrie, au XIII ${ }^{\mathrm{e}}$ siècle puis, surtout, au début des années 1340, ont donc joué un rôle pionnier. On mesure aussi l'importance du culte de Marguerite pour les Angevins qui régnaient à Budapest depuis 1308. La dernière partie du livre est consacrée à une lecture de la Legenda maior, confrontée à sa source principale, les actes de l'enquête de 1276 (laquelle n'est plus conservée que partiellement aujourd'hui, entre un tiers et la moitié du texte manquant). L'auteure étudie le processus de réécriture du dossier, depuis l'enquête, texte source, vers la Legenda, texte cible (sans doute aurait-il fallu citer et utiliser, sur le plan méthodologique au moins, le livre pionnier de Monique Goullet, Écriture et réécriture hagiographiques, Turnhout, 2006). Conformément au phénomène désigné par André Vauchez comme "invasion mystique ", la sainte est devenue fiancée du Christ, ce qu'elle n'était guère au XIII ${ }^{\mathrm{e}}$ siècle. On insiste en revanche 
assez peu sur son appartenance à l'ordre dominicain, contrairement à ce que l'on trouve dans les dossiers d'une Agnès de Montepulciano ou d'une Catherine de Sienne.

Le livre est clair, bien bâti, bien informé. Si l'on devait émettre quelques réserves, ce serait sans doute sur la façon dont la Legenda maior est située dans une longue histoire de l'hagiographie. Le sentiment prévaut souvent, en effet, d'une rupture presque absolue entre l'hagiographie mendiante et tout ce qui a précédé (à l'exception des vies de béguines, mais les saintes cisterciennes ne sont guère prises en compte). On trouve l'affirmation suivante: "à partir de la fin du XII siècle, nous observons des changements importants dans l'hagiographie médiévale. Jusqu'alors, l'Église ne révérait comme saints que des chrétiens des premiers siècles. La vénération des saints “modernes" ou contemporains débute après $1150 »$ (p. 33). Affirmation évidemment beaucoup trop tranchée, et pour tout dire insoutenable. Les vies de saints " contemporains" (proches de leurs hagiographes) sont légion avant la fin du XII siècle, depuis saint Martin de Tours jusqu'aux moines clunisiens, en passant par les missionnaires carolingiens, les évêques d'Empire des $\mathrm{x}^{\mathrm{e}}$ et $\mathrm{XI}^{\mathrm{e}}$ siècles ou les ermites et autres saints militants de l'époque dite "grégorienne "... Le XIII ${ }^{e}$ siècle n'a pas tout inventé en matière d'hagiographie... De même, si les miracles acquièrent un nouveau statut dans les procès de canonisation (ils « certifient » les vertus et les mérites), on ne peut dire que la sainteté antérieure au XIII siècle était toujours fondée prioritairement sur l'action thaumaturgique des saints. Beaucoup de textes pourraient être convoqués : contentons-nous de mentionner Odon de Cluny qui, au $\mathrm{x}^{\mathrm{e}}$ siècle, dans les vitae de Grégoire de Tours et de Géraud d'Aurillac (saint «contemporain » et miles de surcroît), minimise les miracles en rappelant que ni la Vierge ni Jean Baptiste n'en ont accompli. Un autre désaccord porte sur la scène de la mort du saint dans les textes hagiographiques (p. 296) : le "saint trépas » deviendrait " un élément indispensable » «à partir du début du XIve siècle ». Or la lecture des vitae du haut Moyen Âge et de l'époque féodale montre bien qu'elle est déjà essentielle, quantitativement aussi bien que qualitativement. Reste la question de la théologie du miracle dans la Legenda maior. Quelques pages très stimulantes suggèrent que Garin (s'il est bien l'auteur de BHL 5331) a fait passer dans ses récits l'enseignement de Thomas d'Aquin sur les miracles. Le peu de place laissé à la démonstration de cette thèse ne permet pas de rendre celle-ci pleinement convaincante et les textes mis en avant peuvent légitimement éveiller un certain scepticisme. L'affirmation que c'était Dieu qui agissait à travers Marguerite, l'« exaltation de la foi catholique », la " volonté de susciter la dévotion dans le cœur des fidèles ", tous ces thèmes sont assurément présents chez Thomas (Somme théologique, qu. 178, $\left.\mathrm{II}^{\mathrm{a}}, \mathrm{II}^{\mathrm{ae}}\right)$, mais ils le sont aussi dans des centaines voire dans des milliers de textes hagiographiques antérieurs. Nous avons là des topoï tellement répandus qu'ils sont constitutifs d'un genre et d'un discours. On aurait donc aimé une démonstration plus serrée.

5 Tout compte fait, cet excellent livre constitue à la fois une introduction au dossier hagiographique de Marguerite de Hongrie, à l'hagiographie dominicaine des XIII et XIV siècles et un bel exemple d'étude des processus de réécriture dans l'hagiographie de la fin du Moyen Âge. Ce n'est pas peu. 


\section{AUTEURS}

\section{PATRICK HENRIET}

École Pratique des Hautes Études, Paris. 